\title{
The small amplitude of density turbulence in the inner solar wind
}

\author{
S. R. Spangler \\ University of Iowa, USA
}

Received: 24 September 2001 - Accepted: 4 December 2001

\begin{abstract}
Very Long Baseline Interferometer (VLBI) observations were made of radio sources close to the Sun, whose lines of sight pass through the inner solar wind (impact parameters $16-26 R_{\odot}$ ). Power spectra were analyzed of the interferometer phase fluctuations due to the solar wind plasma. These power spectra provide information on the level of plasma density fluctuations on spatial scales of roughly one hundred to several thousand kilometers. By specifying an outer scale to the turbulence spectrum, we can estimate the root-mean-square (rms) amplitude of the density fluctuations. The data indicate that the rms fluctuation in density is only about $10 \%$ of the mean density. This value is low, and consistent with extrapolated estimates from more distant parts of the solar wind. Physical speculations based on this result are presented.
\end{abstract}

\section{Introduction}

This paper will deal with observations of the plasma, and specifically plasma turbulence in the inner solar wind. More precisely, I will discuss the results of radio propagation, remote sensing observations of the solar wind at heliocentric distances of 16 to $26 \mathrm{R}_{\odot}$. A more complete discussion and presentation of additional results is given in Spangler et al. (2002). Spangler et al. (2002) also gives more information concerning the observations, data reduction, and analysis.

\subsection{Motivation for the observations}

The ultimate goal of these observations is to contribute to the verification or falsification of models for the acceleration and heating of the solar wind. We are specifically interested in testing models in which plasma turbulence plays a role, by heating and accelerating the solar wind. The ideal observational test of turbulence-driven models of the solar wind

Correspondence to: S. R. Spangler

(srs@astro.physics.uiowa.edu) would be to measure the intensity and spectral characteristics of magnetic field and plasma velocity fluctuations in the part of space between the coronal base and a heliocentric distance of about $60 \mathrm{R}_{\odot}$. Such measurements are not available, and will not be available until the advent of Solar Probe. In the meantime, one of the few techniques available to us is radio remote sensing measurements, which furnish information on the intensity and spectral characteristics of density fluctuations.

The specific approach in this paper is as follows:

1. We use radio remote sensing observations (described further in the next section) to measure the properties of density fluctuations as a function of heliocentric distance $r$.

2. We assume that the density fluctuations serve as a proxy for the dynamically more interesting magnetic field fluctuations and velocity fluctuations. This means that the normalized amplitude of the density fluctuations is assumed equal to that of the magnetic field fluctuations, and the spatial power spectra are assumed to be the same. Further discussion of this controversial (and possibly wrong!) assumption is given in Sect. 4.1 below.

3. The inferred level of turbulence can be compared with that necessary for substantial dynamical and thermodynamical effects in accelerating and heating the solar wind.

The observations utilized in this paper provide information on the solar wind plasma as close as $16 \mathrm{R}_{\odot}$ from the Sun. This closest perihelion distance was chosen for radio astronomical technical reasons, in that analysis of interferometer phase scintillation data is most convenient in the heliocentric distance range $16-30 \mathrm{R}_{\odot}$. Since a number of independent observations utilizing ultraviolet spectroscopy indicate substantial coronal heating much closer to the Sun (e.g. Kohl et al., 1999; Marsch, 1999) at heliocentric distances of $1-3 \mathrm{R}_{\odot}$, the utility of observations further out can be questioned. There 
are three reasons why I believe radio astronomical observations of plasma at heliocentric distances of $\simeq 16 R_{\odot}$ are important. First, the aforementioned evidence of ion heating in the corona, presumed to be due to plasma turbulence, is derived from spectroscopic line profiles. For a consistent description of coronal processes, measurement of the turbulence itself would be highly desirable.

Second, there is the question of what I term the vestigium undarum, or relic of the waves which are not dissipated close to the Sun in ion heating. Processes in nature are not $100 \%$ efficient, and it is reasonable to expect some reasonable portion of the wave flux, injected at the coronal base, to survive to $16 R_{\odot}$ and beyond. Radio scintillation measurements might detect the vestigium. Finally, while evidence has been put forward indicating that the fast solar wind is rapidly accelerated close to the Sun, it seems clear that the slow solar wind continues to accelerate out to $30 \mathrm{R}_{\odot}$ and perhaps beyond (Sheeley et al., 1997; Schwenn et al., 1981).

Thus, the processes responsible for acceleration of the slow solar wind, which presumably include the effects of turbulence, are distributed over a large volume of space. As discussed in Spangler and Sakurai (1995), radio propagation measurements are generally dominated by the slow speed solar wind, so radio observations are well suited to provide diagnostics of this plasma.

\subsection{The physical basis of phase scintillations}

The physical basis of interferometer phase scintillations due to turbulence in the solar wind has been discussed previously by Spangler and Sakurai (1995) and numerous reports exist on the general phenomenon of interplanetary scintillations (e.g. Coles, 1978; Bourgois, 1993). The relationship between plasma turbulence and radio astronomical measurements is illustrated in Fig. 1. In plasma turbulence, there will be fluctuations in the plasma density. Since the radio refractive index is proportional to the plasma density, there will be corresponding fluctuations in the refractive index. The phase of the radio wave at each antenna is proportional to the line of sight integral of the refractive index from the antenna to the source of the radio waves. An interferometer measures the difference of the radio wave phase at the two antennas. When the interferometer observes a source through a moving medium with index of refraction variations, the interferometer phase varies as a random function of time. Roughly speaking, the greater the amplitude is of the density fluctuations (i.e. the more intense the turbulence), the larger the phase fluctuations will be. The faster the medium is moving, the more rapid these variations will be.

\section{Measurements of VLBI phase scintillations}

The measurements presented here result from interferometric observations made with radio telescopes of the Istituto di Radioastronomia at Medicina and Noto in Italy. In addition, observations were made with the Matera (Italy) antenna of

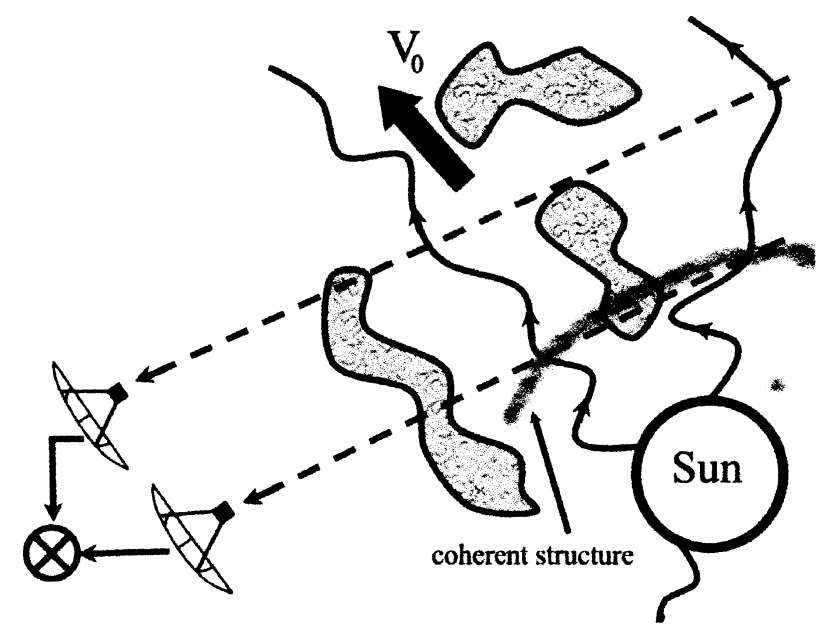

Fig. 1. An illustration of how density fluctuations in a turbulent plasma, causing radio refractive index variations, produce stochastic perturbations in the phase of a radio interferometer. Motion of the turbulent plasma then causes temporal fluctuations in the interferometer phase. Figure taken from Spangler et al. (2002).

the Agenzia Spaziale Italiana, and the Wettzell (Germany) antenna of the Bundesamt für Kartographie und Geodäsie. These observations were undertaken as a program of the Istituto di Radioastronomia of the Consiglio Nazionale delle Ricerche, located at Bologna. Observations were made on 24 and 25 September, and 15 October 1998. The observational sessions ranged from 6 to $9 \mathrm{~h}$ in duration. The observations were made (depending on the day and the telescopes participating) at either 8.38 and $2.26 \mathrm{GHz}$, or $4.98 \mathrm{GHz}$. Observing scans on a source were $13 \mathrm{~min}$ in duration, and for each interferometer baseline a phase measurement was recorded each $2.0 \mathrm{~s}$. The integration time was also $2.0 \mathrm{~s}$. The interferometer baselines varied in length from a minimum of about $200 \mathrm{~km}$, to almost $1000 \mathrm{~km}$. The baseline lengths are important control parameters because phase scintillations are produced mainly by irregularities with size scales of the order of the baseline length.

Measurements were made of five sources viewed through the inner solar wind. The distance of closest approach of the line of sight (referred to as the "impact parameter") varied from 16.5 to $25.3 \mathrm{R}_{\odot}$. Since the solar wind plasma is densest at the point of closest approach to the Sun, the plasma in the vicinity of the impact parameter makes the dominant contribution to the radio propagation phenomenon. One can roughly think of phase scintillation measurements as diagnosing the plasma at a heliocentric distance equal to the impact parameter. In addition to the five sources observed through the inner solar wind, observations were made of another source at an impact parameter of $62.7 \mathrm{R}_{\odot}$.

Interferometer phase fluctuations are analyzed by calculating the power spectrum of a phase fluctuation time series, and then fitting the spectrum to a theoretical model (Spangler et al., 2002). This phase power spectrum is related, via a propagation model, to the spatial power spectrum of the density 
fluctuations in the solar wind. We model that spatial power spectrum with the following expression

$$
P_{\delta n}(k)=C_{N}^{2} k^{-\alpha}
$$

The spectrum is defined on the wave number range $2 \pi / l_{o} \leq$ $k \leq 2 \pi / l_{i}$. In Eq. (1), $k$ is the spatial wave number of the density fluctuations, $\alpha$ is the spectral index of the power law spectrum, and $C_{N}^{2}$ is the coefficient which determines the level of the density fluctuations. This parameter $C_{N}^{2}$ is directly proportional to the variance of the density fluctuations and is a function of the heliocentric distance $r$. The outer and inner scales of the turbulence are given by $l_{o}$ and $l_{i}$, respectively. These correspond to the largest and smallest scales present in the turbulence.

Measurement and analysis of the phase fluctuations yield the scattering measure $S M$, which is the path integral of $C_{N}^{2}$ through the solar wind,

$$
S M \equiv \int_{\mathrm{LOS}} C_{N}^{2}(s) d s=C_{N 0}^{2} L_{e f f} \simeq C_{N 0}^{2}\left(\frac{\pi R_{0}}{2}\right) .
$$

A discussion of how the scattering measure is obtained from measurements of the interferometer phase fluctuations is given in Spangler and Sakurai (1995). The variable $s$ is a spatial coordinate along the line of sight. In Eq. (2), $L_{e f f}$ is the thickness of an effective uniform slab of turbulence, and the last expression on the right uses the appropriate value of $L_{\text {eff }}$ for the case in which $C_{N}^{2}$ depends on heliocentric distance as $\propto r^{-4}$. The variable $C_{N 0}^{2}$ is the value of $C_{N}^{2}$ at a heliocentric distance equal to the impact parameter, given by $R_{O}$.

In the observations discussed here, the model fits to the measured phase power spectra were not sensitive to $l_{o}$ and $l_{i}$. The principal quantities retrieved were $C_{N 0}^{2}$ and the solar wind flow speed $V_{0}$. The outer scale emerges as an important parameter in the analysis of Sect. 3.2, where it will have to be specified on the basis of independent considerations.

\section{Observational results}

In this section, I present selected results from this set of observations. The results presented will deal solely with the amplitude of the density fluctuations.

\subsection{The evolution of $C_{N}^{2}$ with heliocentric distance}

Fitting of the observed phase power spectra with a theoretical model allows for the retrieval of $C_{N 0}^{2}$. Since different observations will have different impact parameters and probe the solar wind at different heliocentric distances, an extensive observational program with observations of many sources can establish $C_{N}^{2}(r)$. Our results from several observing sessions over many years are shown in Fig. 2. All open symbols are results presented by Spangler and Sakurai (1995) and Spangler et al. (1996). All measurements are from VLBI phase scintillation measurements, except the open triangles. Those data are taken from in situ measurements by the Helios

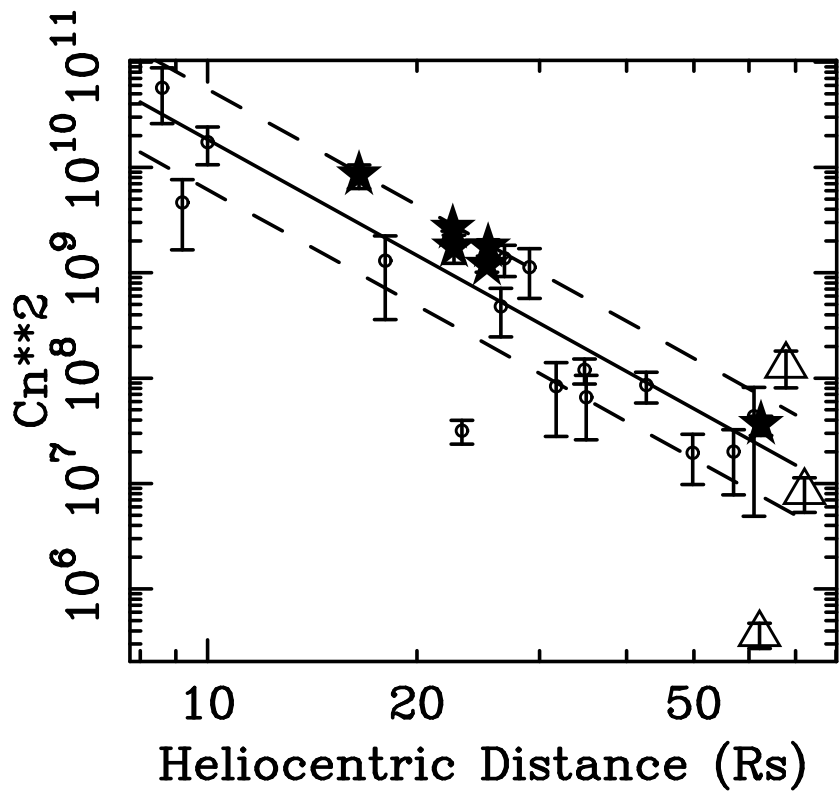

Fig. 2. Empirical measurement of $C_{N}^{2}(r)$. Open symbols display measurements from earlier investigations, reported in Spangler and Sakurai (1995) and Spangler et al. (1996). Open triangles represent values deduced from in situ measurements made by the Helios spacecraft. Large solid stars represent results from the recent set of observations. The solid line represents an empirical $C_{N}^{2}(r)$ relationship reported by Spangler et al. (1996) and given in Eq. (3). The dashed lines indicate the bounds of natural solar wind variability of roughly a factor of three above and below the relation (3). The units of $C_{N}^{2}$ are $\mathrm{m}^{-20 / 3}$. The figure is taken from Spangler et al. (2002).

spacecraft at its perihelion. The spacecraft measurements were presented by Marsch and Tu (1990); the extraction of $C_{N}^{2}$ values from the published spacecraft power spectra is described in Sect. 4.1 of Spangler and Sakurai (1995). The solid stars represent new results from the Italian VLBI observations in the fall of 1998.

Of the Helios measurements, the upper two plotted points (triangles) represent measurements in the slow speed solar wind, while the lower symbols represent high speed wind characteristics. It was on the basis of these measurements that Spangler and Sakurai (1995) argued that the slow speed wind will typically dominate radio scintillation measurements.

The solid line in Fig. 2 represents a least-squares-fit empirical relationship for $C_{N}^{2}(r)$, which was made to all data except a low point at $9 \mathrm{R}_{\odot}$ observed through a polar coronal hole, an anomalous but seemingly valid measurement at $23 \mathrm{R}_{\odot}$, and the in situ measurement in the high speed solar wind (Spangler et al., 1996)

$C_{N}^{2}(r)=1.8 \times 10^{10}\left(r / 10 \mathrm{R}_{\odot}\right)^{-3.66} \mathrm{~m}^{-20 / 3}$.

The dashed lines represent bounding curves at a factor of three above and below the least-squares-fit relationship, and are meant to convey the natural variability in the characteristics of the solar wind. 
The new measurements, which I believe to be more precise than those previously presented in Spangler and Sakurai (1995) and Spangler et al. (1996), contribute to a fuller specification of the $C_{N}^{2}(r)$ relation. Since they fall within the "box" determined by the dashed lines in Fig. 2, they may be considered consistent with and supportive of that relationship. Further observations of this type, which would more densely populate Fig. 2, could contribute to a better understanding of the spatial evolution of solar wind turbulence, as well as furnishing a diagram which would be useful in detecting interplanetary transients and their associated enhanced turbulence.

\subsection{Measuring $\sigma_{n}$ of the density fluctuations}

The relationship shown in Fig. 2 can be used to determine the dimensionless amplitude, or level of the plasma density fluctuations. To do this, we employ some observational techniques applied to the somewhat similar turbulence in the interstellar medium of our galaxy.

The parameter $C_{N}^{2}$ is a measure of the fluctuations in the plasma density. The total inventory of plasma along the line of sight is given by a similar parameter termed the emission measure, and defined as

$$
E M \equiv \int_{\mathrm{LOS}} n_{e}^{2}(s) d s=n_{e 0}^{2} L_{e f f} \simeq n_{e 0}^{2}\left(\frac{\pi R_{0}}{2}\right)
$$

The emission measure is a quantity of considerable interest in astronomy because it is often directly related to measureable quantities, such as the brightness of emission lines. In the present application to the solar wind, $n_{e 0}$ indicates the plasma density at the point of closest approach to the Sun.

A comparison of the scattering measure and the emission measure can be used to extract a parameter determined by the modulation index of the turbulence $\epsilon$ and the outer scale of the turbulence defined above. The modulation index is defined as

$\epsilon^{2} \equiv\left\langle(\delta n)^{2}\right\rangle / \bar{n}^{2}$

The density modulation index $\epsilon$ is referred to as the "mean fractional density fluctuation" by Woo et al. (1995). The relation between $S M$ and $E M$ is then (Spangler and Cordes, 1998)

$S M=0.181\left[\frac{\epsilon^{2}}{\left(1+\epsilon^{2}\right) l_{0}^{2 / 3}}\right] E M$.

Equation (6) is valid for the case of a Kolmogorov spectrum, for which $\alpha=11 / 3$. The spectral index of the power spectrum determines the multiplicative coefficient outside the square brackets, as well as the power of $l_{o}$ in the denominator of the expression within the brackets. In the solar wind context, we measure the scattering measure via observations described in Sect. 2, and use models for the density profile in the slow solar wind (see Eq. (11) below) to compute the emission measure. Thus we determine the quantity in square brackets in Eq. (6).
The outer scale of the turbulence in this part of space $\left(16 \mathrm{R}_{\odot} \leq r \leq 26 \mathrm{R}_{\odot}\right)$ is not known with great precision, but must be of the order of 1 solar radius. The outer scale should be considerably less than the heliocentric distance of $16-26 \mathrm{R}_{\odot}$. It may be seen from LASCO coronagraph images that the transition from fast to slow solar wind occurs on scales smaller than this. I have adopted $l_{o}=1 \mathrm{R}_{\odot}$ in the analysis described here. In any case, Eq. (6) shows that the dependence on the outer scale is not strong. We can then extract the quantity of interest, which is the modulation index $\epsilon$.

When Eq. (6) is used with the data shown in Fig. 2, the values for $\epsilon$ depend on the source being analyzed and the independent model for the background plasma density $n_{e}(r)$ which is employed. The range of the values we obtain is 0.064 to 0.15 ; a representative average is 0.10 . No evidence is seen for a dependence on heliocentric distance. This result is at mild variance with the interstellar medium case discussed by Spangler and Cordes (1998), in which a value of $\epsilon \simeq 1$ was consistent with measurements and plausible values for the outer scale. There was no compelling case for any of the lines of sight through the interstellar medium in Cygnus for values of $\epsilon$ as small as those discussed here for the inner solar wind.

From this analysis we then conclude that the density modulation index seems small, and that weak turbulence in the inner solar wind seems to be indicated.

\section{Significance of the observations}

In this section, I discuss the physical significance of the result presented above, that the root-mean-square density fluctuation in the solar wind between 16 and $26 \mathrm{R}_{\odot}$ is of the order of $10 \%$.

4.1 Density fluctuations as a proxy for magnetic fluctuations

As mentioned in the Introduction, theories of magnetohydrodynamic turbulence indicate that the energy density is primarily or exclusively comprised of magnetic and kinetic energy terms. The theoretically, most easily interpreted measurements would, therefore, be of magnetic and velocity fluctuations, rather than the density fluctuations which are revealed by radio scintillation observations. The radio measurements would be most useful if the density fluctuations can serve as a proxy for magnetic field and velocity fluctuations. To make the argument that this proxy relationship exists, we need theoretical results which are supported by in situ spacecraft measurements. These theoretical and observational results can also determine the exent of the similarity between density and magnetic field fluctuations. The relevant theoretical results for this suggestion are cited below:

1. Zank and Matthaeus (1992) showed that within the context of quasi two-dimensional turbulence, density fluctuations did not arise at first order in the turbulent ampli- 
tude, but did arise at second order. This would suggest that

$$
(\delta n / \bar{n})=A\left(\delta b / B_{0}\right)^{2},
$$

where $A$ is a constant of order unity. In this case, large amplitude magnetic turbulence (and velocity turbulence) could exist with small amplitude density fluctuations. The measurement of small-amplitude density fluctuations would be of uncertain physical interpretation, and the density fluctuations would be a poor proxy for the important energy-containing fluctuations. A requirement for this result (Eq. 7) is homogeneity of the background plasma, i.e. no spatial gradients in the mean plasma parameters such as density and vector magnetic field.

2. Bhattacharjee et al. (1998) showed that if the background plasma had zero order spatial gradients, instead of being spatially homogeneous, then first order density fluctuations accompany magnetic field and velocity fluctuations,

$(\delta n / \bar{n}) \simeq\left(\delta b / B_{0}\right)$

provided that the inhomogeneity of the background plasma is sufficiently pronounced. In Eq. (8), it is to be understood that $\delta b$ is the transverse (to the large scale field) turbulent amplitude, i.e. the quadratic sum of the field components perpendicular to $\boldsymbol{B}_{\mathbf{0}}$. In this case, small-amplitude density fluctuations would imply similarly small-amplitude magnetic field fluctuations, and density variations would be a good proxy for magnetic turbulence. Figure 6 of Bhattacharjee et al. (1998) shows that even weak spatial gradients are sufficient to insure first order density fluctuations.

3. Given the aforementioned considerations, one can invoke the known spatial structuring of the solar wind, such as that due to the stream structure, for example, to argue that the proxy-like scaling of Eq. (8) will hold for the solar wind. I then propose that the dimensionless amplitude of the density fluctuations tells us the corresponding dimensionless amplitude of the magnetic fluctuations.

The suggestion that the normalized density fluctuations are directly proportional to those of magnetic field is a controversial one that is disputed by other workers in the field. An example of a study which is cited as contrary to the relation (8) is that of Tu and Marsch (1994), which presented a comparison of fluctuations in density, magnetic field and plasma velocity. Tu and Marsch (1994) compared the observed correlation coefficient between pressure and turbulent Mach number or Alfvénic Mach number (measures of the amplitude of velocity or magnetic field fluctuations, respectively) with the expected value for simplified models of MHD turbulence. The turbulent Mach number is defined as
$M \equiv \delta v / c_{s}$, where $\delta v$ is the rms velocity fluctuation and $c_{s}$ is the sound speed. The Alfvénic Mach number is similarly defined as $\delta v / V_{A}$, with $V_{A}$ the Alfvén speed. The simplified models for the solar wind fluctuations which were considered included nearly incompressible turbulence, specific wave modes, and pressure-balanced structures. The observational results defied unambiguous interpretation in terms of any of the theoretical models. The obscure nature of the density fluctuations can be considered as a demonstration of the futility of using these fluctuations as diagnostics of other properties of the turbulence.

While this objection is a plausible one, and should be seriously considered by workers in the field, I do not believe that it necessarily invalidates the use of density fluctuations as a proxy for the level of magnetic field and velocity fluctuations. For the proxy hypothesis to be valid, it is only necessary that the variances of the fluctuations in density and magnetic field be mutually proportional, not that a high or even stationary degree of correlation exist between them. In support of this suggestion, we cite the work of Bavassano and Bruno (1995), which directly compared the root-mean-square density fluctuation with the turbulent Mach number. The data for the study of Bavassano and Bruno (1995) were taken from the Helios spacecraft, which approached to approximately $62 \mathrm{R}_{\odot}$ from the Sun. For Alfvénic turbulence in a plasma with $\beta=1, M=M_{A}=\delta b / B_{0}$. The finding of Bavassano and Bruno (1995) was that the in situ measured values of the normalized density fluctuations were much closer to being proportional to $M$ than $M^{2}$, and thus, serve as proxies for the magnetic and velocity fluctuations in the sense defined above.

Thus, if the correlation determined by Bavassano and Bruno (1995) continues to be valid at heliocentric distances closer than $62 \mathrm{R}_{\odot}$, a probable situation in my opinion, the results of Sect. 3.2 would then indicate that the dimensionless amplitude of magnetic field and velocity fluctuations at $16-26 \mathrm{R}_{\odot}$ is also of the order of $10 \%$.

\subsection{Astrophysical significance of a small density modula- tion index}

I now consider the quantitative significance of this small turbulence amplitude. The main interest here is whether the small values for the turbulent amplitude can be reconciled with the requirements of wave-driven solar wind models.

For the sake of argument in the subsequent sections, I assume the validity of Eq. (8). In this case, the radio scintillation observations allow for an estimate of the turbulent magnetic field in the inner solar wind to be extracted, and we can use the procedures and arguments employed previously in the literature (e.g. Hollweg et al., 1982; Sakurai and Spangler, 1994; Mancuso and Spangler, 1999), to assess the viability of wave heating and acceleration mechanisms. The following discussion is also influenced by the arguments of Roberts (1989).

The following calculations will refer to a heliocentric distance of $16 \mathrm{R}_{\odot}$, the closest point to the Sun probed by the 
observations of Spangler et al. (2002), and at which $0.060 \leq$ $\frac{\delta n}{\bar{n}}, \frac{\delta b}{B_{0}} \leq 0.12$.

If the magnetic turbulence is envisioned as composed of Alfvén waves, there is an associated wave energy flux given by (Mancuso and Spangler, 1999, Eq. (10), originally due to Hollweg, 1973)

$S_{w}=\frac{\left\langle(\delta b)^{2}\right\rangle}{8 \pi}\left(2 V_{A}+3 V\right)$,

where $V$ is the bulk solar wind speed. All parameters in Eq. (9) are evaluated at a given heliocentric distance. To calculate the variables in Eq. (9) from our observed measurement of $\delta n / \bar{n}$, we need estimates for $B_{0}, \bar{n}$, and $V$ as a function of heliocentric distance.

For the solar wind magnetic field strength, we use the expression employed by Mancuso and Spangler (2000), which is believed to be valid throughout the region considered here

$B_{0}(r)=5.96\left(r / \mathrm{R}_{\odot}\right)^{-3}+1.43\left(r / \mathrm{R}_{\odot}\right)^{-2} \mathrm{G}$.

Equation (10) is taken from Eq. (7) of Mancuso and Spangler (2000) and converted to more convenient units for the discussion here.

The plasma density is represented by the model of Gibson et al. (1999) for the slow speed solar wind,

$\bar{n}(r)=\left[365\left(r / \mathrm{R}_{\odot}\right)^{-4.31}+3.6\left(r / \mathrm{R}_{\odot}\right)^{-2}\right] \times 10^{5} \mathrm{~cm}^{-3}$.

Finally, the solar wind speed is taken to be given by the following model of Sheeley et al. (1997)

$V^{2}(r)=2 a\left(r-r_{1}\right)$

with $a=3.4 \mathrm{~m} / \mathrm{sec}^{2}$ and $r_{1}=-0.4 \mathrm{R}_{\odot}$.

With these adopted models, the values for solar wind plasma parameters at $16 \mathrm{R}_{\odot}$ are $B_{0}=7.04 \times 10^{-3} \mathrm{G}$, $\bar{n}=1.64 \times 10^{3} \mathrm{~cm}^{-3}, V_{A}=363 \mathrm{~km} / \mathrm{s}$, and $V=279 \mathrm{~km} / \mathrm{s}$. Equation (9) then gives $S_{w}=1.11-4.44 \mathrm{ergs} / \mathrm{s} / \mathrm{cm}^{2}$ for $\delta n / \bar{n}=0.06-0.12$.

Following the ideas of Roberts (1989), we can compare this wave flux with the kinetic energy flux of the bulk solar wind,

$S_{k}=\frac{1}{2} \rho V^{3}$,

where $\rho$ is the solar wind mass density. For the plasma parameters given above, $S_{k}=32.5 \mathrm{ergs} / \mathrm{s} / \mathrm{cm}^{2}$. Thus, at a heliocentric distance of $16 \mathrm{R}_{\odot}$, we estimate that the turbulent energy flux in the slow solar wind is only $3.4 \%$ to $13.7 \%$ of the bulk kinetic energy flux.

Another way of expressing the low value of the turbulent amplitude is by estimating the wave flux at the coronal base, and comparing this with the widely cited values required by wave-driven models for the corona and solar wind. The models require a wave flux of $(2-5) \times 10^{5} \mathrm{ergs} / \mathrm{s} / \mathrm{cm}^{2}$ to match observations. Once again, we employ formulas discussed and utilized in the analysis of coronal Faraday rotation data (Mancuso and Spangler, 1999, and references therein). The Alfvén wave flux at the coronal base can be estimated from plasma parameters $\left(\delta b, V_{A}, V\right.$, etc.) at a heliocentric distance $r$ via (Spangler and Mancuso, 1999, Eqs. 11 and 12)

$S_{W B}=\frac{\left\langle(\delta b)^{2}\right\rangle}{4 \pi V_{A}}\left(V+V_{A}\right)^{2}\left(\frac{r}{\mathrm{R}_{\odot}}\right)^{2} f(r)$,

where all parameters have been defined except $f(r)$, which is a flux tube expansion factor. This factor describes the extent to which the expansion exceeds radial expansion, as in that of a ballooning flux tube. A value of 6.5 has been employed in previous analyses. Although such a value is reasonable for coronal holes, it is probably a major overestimation for regions of slow solar wind. I will employ $f=6.5$ with the understanding that it results in an upper limit to the coronal base flux.

With these assumptions and adoptions of numbers previously used above, we have $S_{W B}=2.68 \times 10^{3}-1.07 \times$ $10^{4} \mathrm{ergs} / \mathrm{s} / \mathrm{cm}^{2}$, where the range corresponds to the allowed range of values of $\delta n / \bar{n}$. These numbers are one to two orders of magnitude lower than the base fluxes of $(2-5) \times$ $10^{5} \mathrm{ergs} / \mathrm{s} / \mathrm{cm}^{2}$ required by wave-driven wind theories. These calculations quantify the weakness of the turbulence in the inner solar wind, and indicate that it is not at a level which is dynamically or thermodynamically significant. Nonetheless, there are three major caveats which need to be appended to the preceding discussion:

1. The base flux to which our calculations have been referred is that required for models of the fast solar wind. The radio propagation observations, which are the basis of this analysis, are primarily diagnostics of the slow solar wind. It is less obvious that wave dissipation is required to account for the properties of the slow solar wind, so the comparison made here might be meaningless.

2. Even if similar base fluxes are injected in open and closed magnetic field regions, there is no guarantee that a substantial fraction of the wave flux survives to $16 R_{\odot}$, rather than being completely damped within a couple of solar radii of the solar surface. This point of view has been emphasized to me by C.Y. Tu in private conversations. My response is that such an explanation of the data would require 90 to $99 \%$ efficiency of wave damping inside $16 \mathrm{R}_{\odot}$. This issue is essentially that of the vestigium discussed in Sect. 1.1.

3. A crux of the analysis has been the assumption of equal dimensionless magnetic and density fluctuations given in Eq. (8). It needs to be acknowledged that other remote sensing observations of the inner solar wind have been cited as evidence for $\delta b / B_{0} \gg \delta n / \bar{n}$. Hollweg et al. (1982) analyzed variations in Faraday rotation measure and radio phase of a spacecraft transmitter signal. Faraday rotation is proportional to the path integral of the plasma density and line-of-sight component of the magnetic field, and thus, contains information on the magnetic field and its fluctuations. The phase measurement is sensitive to the path integral of density alone. 
Hollweg et al. (1982) examined the minimalist possibility that both Faraday rotation and phase variations could be accounted for by density fluctuations in a uniform magnetic field. They found that this extreme and nearly unphysical model could be excluded, since the required magnetic field would have to be about a factor of 5 higher than indicated by independent estimates. This result indicated that magnetic field fluctuations made an important and possibly dominant contribution to the rotation measure fluctuations. Indeed, Hollweg et al. (1982) then proceeded to analyze their data with model equations which attributed all of the Faraday rotation measure fluctuations to magnetic field variations in an incompressible plasma, as would be the case if the turbulence consisted of ideal Alfvén waves.

While the discussion of Hollweg et al. (1982) rules out hypothetical turbulence in which only density fluctuations are present, it cannot be used to exclude all models in which $\delta n / \bar{n} \sim \delta b / B_{0}$. The reason for this can be understood via reference to Eq. (17) of Hollweg et al. (1982), which gives an expression for the rotation measure variance. This expression contains three contributions, one due to magnetic field fluctuations in the mean density of the plasma, the second due to density fluctuations in the mean magnetic field, and a third dependent on the amplitude of both magnetic and density fluctuations, as well as their correlation. The case of exclusively density fluctuations examined and rejected by Hollweg et al. (1982) had only one of these three terms contributing. The fact that an admissible turbulence and plasma model could be constructed with only magnetic field fluctuations in a constant density plasma does not exclude the possibility that turbulence with $\delta n / \bar{n} \sim \delta b / B_{0}$ could also be compatible with the data of Hollweg et al. (1982). To determine if this is the case would require a reanalysis of the data in Hollweg et al. (1982) to see if compatibility could be achieved with turbulence describable by Eq. (8), and plausible sets of solar wind plasma properties. Such an analysis would be highly worthwhile and should be done, but is beyond the scope of the present paper.

\section{Conclusions and exhortations}

The following represent the main points of this paper:

1. The techniques of radio astronomical propagation measurements provide information about density fluctuations throughout the inner heliosphere, i.e. regions interior to those which have been explored by spacecraft. The technique of choice in this presentation, fluctuations in the phase of a Very Long Baseline Interferometer, provide some novel features relative to other radio propagation measurements, such as intensity scintillations.
2. Analysis of the data yields estimates of the fractional density fluctuations at heliocentric distances of 16 to $26 \mathrm{R}_{\odot}$. These values are relatively low, of the order of 6 to $15 \%$, with an average of about $10 \%$. If the normalized density turbulence is proportional to the normalized magnetic turbulence (admittedly a big "if"; see the discussion in Sect. 4.1 above), then the turbulence in the inner solar wind is relatively weak and perhaps incapable of playing a significant role in the dynamics or thermodynamics of the solar wind.

3. Basic plasma physics research on the nature of density fluctuations which arise in a turbulent plasma would seem to be very worthwhile, the substantial existing literature in this area notwithstanding. Solid results from such a theory would allow us to interpret the radio scintillation observations in a more mature way. A specific topic identified for attention in this research would be the validity or non-validity of the proxy relationship between density and magnetic field fluctuations.

4. The justification for further work in radio remote sensing of the inner solar wind (including the theoretical work in point \# 3) is that radio propagation studies of the inner solar wind will remain important for heliospheric physics in the future. The deepest penetration of the heliosphere by a definite future spacecraft will be that of the Solar Orbiter, planned for the time period 20082013 , and with a perihelion distance of $45 \mathrm{R}_{\odot}$. Information on the solar wind plasma closer to the Sun will require remote sensing measurements, and radio propagation studies will remain one of the most important.

Acknowledgements. This research was supported at the University of Iowa by grants ATM96-16721 and ATM99-86887 from the National Science Foundation. The observational program was planned and initiated at the Istituto di Radioastronomia of the Italian Consiglio Nazionale delle Ricerche at Bologna. I wish to acknowledge my colleagues M. Bondi and F. Mantovani, who have participated in this project.

\section{References}

Bavassano, B. and Bruno, R.: Density Fluctuations and Turbulent Mach Numbers in the Inner Solar Wind, JGR, 100, 9475, 1995.

Bhattacharjee, A., Ng, C. S., and Spangler, S. R.: Weakly Compressible Magnetohydrodynamic Turbulence in the Solar Wind and the Interstellar Medium, Ap. J., 494, 409, 1998.

Bourgois, G.: Interplanetary Turbulence as Studied by Random Propagation Methods, in: Spatio-Temporal Analysis for Resolving Plasma Turbulence, ESA Publication WPP-047, 1993.

Coles, W. A.: Interplanetary Scintillation, Space Sci. Rev., 21, 411, 1978.

Gibson, S. E., Fludra, A., Bagenal, F., Biesecker, D., del Zanna, G., and Bromage, B.: Solar Minimum Streamer Densities and Temperatures Using Whole Sun Month Coordinated Data Sets, JGR, 104, 9691, 1999.

Hollweg, J. V.: Alfvén Waves in a Two Fluid Model of the Solar Wind, Ap. J. 181, 547, 1973. 
Hollweg, J. V., Hollweg, J. V., Bird, M. K., Volland, H., Edenhofer, P., Stelzried, C. T., and Seidel, B. L.: Possible Evidence for Coronal Alfvén Waves, JGR, 87, 1, 1982.

Kohl, J. L., Esser, R., Cranmer, S. R., Fineschi, S., Gardner, L. D., Panasyuk, A. V., Strachan, L., Suleiman, R. M., Frazin, R. A., and Noci, G.: EUV Spectral Line Profiles in Polar Coronal Holes from 1.3 to $3.0 \mathrm{R}_{\odot}$, Ap. J. 510, L59, 1999.

Mancuso, S. and Spangler, S. R.: Coronal Faraday Rotation Observations: Measurements and Limits on Plasma Inhomogeneities, Ap. J. 525, 195, 1999.

Mancuso, S. and Spangler, S. R.: Faraday Rotation and Models for the Plasma Structure of the Solar Corona, Ap. J. 539, 480, 2000.

Marsch, E.: Cyclotron Heating of the Solar Corona, Astrophysics and Space Science, 264, 63, 1999.

Marsch, E. and Tu, C. Y.: Spectral and Spatial Evolution of Compressible Turbulence in the Inner Solar Wind, JGR, 95, 11 945, 1990.

Roberts, D. A.: Interplanetary Observational Constraints on Alfvén Wave Acceleration of the Solar Wind, JGR 94, 6899, 1989.

Sakurai, T. and Spangler, S. R.: The Study of Coronal Plasma Structures and Fluctuations with Faraday Rotation Measurements, Ap. J. 434, 773, 1994.

Sheeley, N. R., Wang, Y. M., Hawley, S. H., Brueckner, G. E., Dere, K. P., Howard, R. A., Koomen, M. J., Korendyke, C. M., Michels, D. J., Paswaters, S. E., Socker, D. G., St. Cyr, O. C., Wang, D., Lamy, P. L., Llebaria, A., Schwenn, R., Simnett,
G. M., Plunkett, S., and Biesecker, D. A.: Measurement of Flow Speed in the Corona between 2 and $30 \mathrm{R}_{\odot}$, Ap. J. 484, 472, 1997.

Schwenn, R., Mühlhäuser, K. H., Marsch, E., and Rosenbauer, H.: Two States of the Solar Wind at the Time of Solar Activity Maximum, Solar Wind 4, (Ed) Rosenbauer, H., p. 126, 1981.

Spangler, S. R. and Cordes, J. M.: VLBI Measurements of Plasma Turbulence Associated with the Cygnus OB1 Association, Ap. J. 505, 766, 1998.

Spangler, S. R. and Sakurai, T.: Radio Interferometer Observations of Solar Wind Turbulence from the Orbit of Helios to the Solar Corona, Ap. J. 445, 999, 1995.

Spangler, S. R., Sakurai, T., Coles, W. A., Grall, R. R., and Harmon, J. K.: Radio Interferometer Observations of Turbulence in the Inner Solar Wind, Solar Wind 8, AIP Conf. Proc \# 382, 265, 1996.

Spangler, S. R., Kavars, D. W., Kortenkamp, P. S., Bondi, M., Mantovani, F., and Alef, W.: Very Long Baseline Interferometry Measurements of Turbulence in the Inner Solar Wind, Astron. \& Astrophys., in press, 2002.

Tu, C. Y. and Marsch, E.: On the Nature of Compressive Fluctuations in the Solar Wind, JGR, 99, 21 481, 1994.

Woo, R., Armstrong, J. W., Bird, M. K., and Pätzold, M.: Variation of Fractional Electron Density Fluctuations Inside $40 \mathrm{R}_{\odot}$ Observed by Ulysses Ranging Measurements, GRL, 22, 329, 1995.

Zank, G. P. and Matthaeus, W. H.: The Equations of Reduced Magnetohydrodynamics, J. Plasma Phys., 48, 85, 1992. 